\title{
Evaluation of Neoadjuvant Chemotherapy in Treatment of Muscle Invasive Urothelial Bladder Tumors in Upper Egypt
}

\author{
Mohamed A. Khateeb ${ }^{1}$, Diaa A. Hameed ${ }^{1}$, Ahmed M. Moeen ${ }^{1}$, Tareq Salah ${ }^{2}$, Hosney \\ M. Behnsawy ${ }^{1}$, Yasser M. Abdelsalam ${ }^{1}$, Samir Shehata ${ }^{2}$, Magdi A. El-Akkad ${ }^{1}$ \\ ${ }^{1}$ Urology University Hospital, Faculty of Medicine, Assiut University, Assiut, Egypt; ${ }^{2}$ Clinical \\ Oncology and Nuclear Medicine Department, Faculty of Medicine, Assiut University, Assiut, Egypt.
}

Background: Bladder cancer is a common health problem is Egypt where it is the $3^{\text {rd }}$ common cancer (6.9\%) in both sexes and the $2^{\text {nd }}$ common $(10.7 \%)$ among males. Neoadjuvant chemotherapy has proven benefits in treatment of muscle invasive bladder cancer (MIBC), yet it is still underutilized.

Aim: To study the response to neoadjuvant chemotherapy in patients with MIBC and their attitude towards definitive treatment after completion of neoadjuvant therapy.

Methods: In this prospective study, 85 patients with MIBC were recruited between September 2013 and September 2014. They were scheduled to receive three cycles of gemcitabine $\left(1000 \mathrm{mg} / \mathrm{m}^{2}\right.$, on days 1 and 8$)$ plus cisplatin $\left(75 \mathrm{mg} / \mathrm{m}^{2} \mathrm{on}\right.$ day 1) or carboplatin (AUC=5 on day 1) in patients with impaired renal function prior to definitive treatment.

Results: Sixty-seven patients were evaluable for response and toxicity. The majority (79\%) were males and their median age was 61 years (range: $38-84$ ). The initial T stage was T3 or T4 in $72 \%$ of patients. Complete response was documented in $6(9 \%)$ patients, partial response in $41(61.2 \%)$, stationary disease in $5(7.5 \%)$ and progressive disease in $15(22.4 \%)$. Grade III and IV toxicities were infrequent (5\%) with no chemotherapy-related mortality. After completion of the treatment, $9(13.4 \%)$ patients were shifted to bladder preservation treatment due to complete radiological response and refusal of surgery.

Conclusion: Neoadjuvant chemotherapy is feasible in MIBC patients in our setting as it gives good clinical response. If offered in a proper way, it doesn't preclude the patients' chances for definitive treatment.

Keywords: Bladder cancer, Neoadjuvant chemotherapy, Bladder preservation, Uro-oncology, Upper Egypt

Corresponding Author: Tareq Salah, M.D.; Clinical Oncology and Nuclear Medicine Department, Faculty of Medicine, Assiut University, Assiut, Egypt; Email: tareqsalah41@yahoo.com

Submitted: 30-June-2017, Revised: 18-September-2017, Accepted: 30-September-2017, Published online: 11-November-2017

\section{INTRODUCTION}

Bladder cancer $(\mathrm{BC})$ is one of the most common cancers worldwide. In Egypt, it is the $3^{\text {rd }}$ common cancer $(6.9 \%)$ in both sexes after liver and breast cancers and the $2^{\text {nd }}$ common $(10.7 \%)$ among males ${ }^{1}$. There is geographical variability in the incidence of $\mathrm{BC}$ which is higher in Upper Egypt (7.4\%) than in Lower Egypt $(5.9 \%)^{1}$. The golden standard for the management of muscle invasive bladder cancer (MIBC) is radical cystectomy with pelvic lymphadenectomy ${ }^{2}$. Despite potentially curative surgery almost half of MIBC patients develop local recurrence and/or distant metastasis which affect their survival ${ }^{3,4}$.

To improve the results of surgery for MIBC, neoadjuvant chemotherapy (NAC) has been explored. Radical cystectomy provides the best local control of the $1^{\text {ry }}$ bladder tumors; however, cancer is a systemic disease and so chemotherapy aims at eradicating the possible micrometastatic disease in addition to its local effect on the $1^{\text {ry }}$ tumor ${ }^{5}$. The rationale behind $\mathrm{NAC}$ is multifactorial. The use of NAC in locally advanced BC permits a rapid in vivo assessment of pathological response ${ }^{6}$. It may reduce the $1^{\text {ry }}$ tumor volume and give the chance for some patients to have a curative surgery. Furthermore, patients may best tolerate chemotherapy while they are in the best possible performance before receiving local treatment whether surgery or radiotherapy that may affect their performance status ? Local treatments may also affect drug delivery to the $1^{\text {ry }}$ tumor by alternating the blood supply. Collectively, NAC has the potential to deliver the drugs more efficiently and at higher doses than in the adjuvant setting in addition to the opportunity to in vivo testing response of the tumor to chemotherapy ${ }^{8}$.

To the best of our knowledge, NAC for MIBC hasn't been studied in our locality till now. The reluctance to use NAC in this setting doesn't seem to be peculiar to Upper Egypt. Although the benefits are well established ${ }^{9}$, the clinical practice is still lagging behind 10. In this study we aimed to evaluate the clinical and radiological responses and the side effects of NAC in a cohort of MIBC patients in Upper Egypt and the attitude of these patients towards definitive treatment following NAC.

\section{METHODS}

This prospective study has been carried out between September 2013 and September 2014 at the Urology and Clinical Oncology departments, Assiut University, Assiut, Egypt. The ethical committee of the Faculty of 
Medicine, Assiut University approved the study. The nature of the study and the expected treatment related toxicity were explained to participants and an informed consent was obtained before enrollment.

Eighty-five patients were enrolled and underwent the following pre-treatment evaluation: detailed history taking and clinical examination; examination under anesthesia and transurethral resection biopsy; chest, abdomen and pelvis computerized tomography (CT) scan or magnetic resonance imaging (MRI) in patients with elevated serum creatinine; bone scan was done if there was clinical suspicion of metastases in the form of bone pain or elevated serum alkaline phosphatase. Laboratory investigations (complete blood count and renal and liver function tests) were done at baseline and before each chemotherapy cycle.

\section{Selection of patients}

Eligible patients were adults ( $>18$ years of age) who have: histopathologically proven urothelial $\mathrm{BC}$; stage $\mathrm{T} 2$, $\mathrm{T} 3$ or T4a, N0, M0; performance status $\leq 1$ by the Eastern Cooperative Oncology Group (ECOG) scale; adequate bone marrow reserve (neutrophil count $>1500 / \mu \mathrm{L}$, platelet count $>100000 / \mu \mathrm{L}$ and hemoglobin $>10 \mathrm{gm} / \mathrm{dL}$ ); creatinine clearance $>60 \mathrm{ml} / \mathrm{min}$ and serum bilirubin and aminotransferases $\leq 2.5$ times the upper limit of normal.

Patients were excluded if they have any of the following: serious or uncontrolled concurrent medical illness, pregnancy or lactation, history of previous cancer, non-urothelial BC, prior systemic chemotherapy or radiotherapy to the bladder, or major surgery within 4 weeks of starting chemotherapy.

\section{Neo-adjuvant chemotherapy (NAC) regimen}

Eligible patients received 3 cycles of NAC that included cisplatin on day 1 and gemcitabine on days 1 and 8.

One hour after intravenous pre-hydration with 1000 $\mathrm{ml}$ normal saline, cisplatin $\left(75 \mathrm{mg} / \mathrm{m}^{2}\right)$ was administered intravenously + $12.5 \mathrm{gm}$ mannitol in $500 \mathrm{ml} 0.45 \%$ normal saline over one hour. This was followed by intravenous post-hydration with $1000 \mathrm{ml}$ normal saline + $10 \mathrm{q} \mathrm{KCI} / \mathrm{L}+8 \mathrm{mEq}$ magnesium sulfate over 1 hour. Cisplatin was substituted with Carboplatin in 4 patients because of grade II renal impairment after starting treatment (creatinine clearance $<60 \mathrm{ml} / \mathrm{min}$ ) or decline in performance status. Carboplatin was administered with dose targeting area under the curve 5 (AUC=5).

Gemcitabine $\left(1000 \mathrm{mg} / \mathrm{m}^{2}\right)$ was administered intravenously in $250 \mathrm{ml}$ normal saline over 30 minute.

\section{Post-treatment evaluation}

History taking, clinical examination and chest, abdomen and pelvis CT scans or MRI were done to evaluate tumor response. The response was determined according to the Response Evaluation Criteria In Solid Tumors (RECIST) version $1.1^{11}$ as follows:

- Complete Response (CR): Disappearance of all target lesions.
- Partial Response (PR): At least a 30\% decrease in the sum of the longest diameters of target lesions.

- Stable Disease (SD): Neither sufficient shrinkage to qualify for PR nor sufficient increase to qualify for progressive disease.

- Progressive Disease (PD): At least a 20\% increase in the sum of the LD of target lesions or the appearance of one or more new lesions.

Toxicity was graded according to the National Cancer Institute Common Terminology Criteria for Adverse Events (NCI-CTCAE) version 4.0 ${ }^{12}$.

\section{Statistical analysis}

Data was analyzed using the Statistical Package for Social Sciences (SPSS) software version 14.0. Chi square test was used to compare categorical data. $\mathrm{P}$ value $<0.05$ was considered significant.

\section{RESULTS}

Sixty-seven out of 85 (79\%) recruited patients were evaluable with a dropout rate of $21 \%$. Three patients discontinued treatment after the $2^{\text {nd }}$ cycle due to severe local symptoms and preferred to undergo surgery immediately. Another 3 patients were excluded due to the discovery of undetected distant metastasis (2 patients) or the development of grade 4 renal impairment (1 patient). One female patient who aged 71 years with pT4a tumor died during the $2^{\text {nd }}$ cycle. Her death was not treatment-related. The other 11 patients were withdrawn from the study because of their preference to receive treatment in other centers near their localities.

Table 1: Characteristics of 67 patients

\begin{tabular}{lll}
\hline \multicolumn{1}{l}{ Age } & No. & \% \\
\hline \multicolumn{1}{l}{ Median (range) } & $61(38-84)$ \\
\hline Sex & & \\
\hline Male & 53 & 79.1 \\
\cline { 2 - 3 } Female & 14 & 20.9 \\
\hline T Stage & & \\
\hline T2 & 19 & 28.4 \\
\hline T3 & 34 & 50.7 \\
\hline T4 & 14 & 20.9 \\
\hline Grade & & \\
\hline Low & 5 & 7.5 \\
\hline Intermediate & 6 & 9 \\
\hline High & 56 & 83.6 \\
\hline Squamous differentiation & & \\
\hline Yes & 33 & 49.3 \\
\hline No & 34 & 50.8 \\
\hline Smoking history & & \\
\hline Active & 51 & 76.1 \\
\hline Passive & 8 & 11.9 \\
\hline None & 8 & 11.9 \\
\hline
\end{tabular}

The baseline characteristics of the evaluable 67 patients are shown in table 1. All patients had no radiological evidence of lymph node metaststses (N0) and no evidence of distant metstases (M0). All active smokers were males and all passive smokers were females. 
The response to NAC was CR in $6(9 \%)$ patients, $\mathrm{PR}$ in $41(61.2 \%)$, SD in $5(7.5 \%)$ and PD in $15(22.4 \%)$. The overall response rate $(\mathrm{CR}+\mathrm{PR}+\mathrm{SD})$ was $77.6 \%$. It differed significantly according to the $\mathrm{T}$ stage of tumors, age of patients and smoking history as shown in table 2 . Younger $(\leq 60$ years) patients, those with T2 and T3 stage and non-smokers had better overall response rate. Among the 8 non-smokers, 5 achieved CR and 3 PR. The other studied variables did not correlate significantly with response to NAC.

Table 2: Variables with significant correlation with the overall response rate

\begin{tabular}{|c|c|c|c|c|}
\hline & No. & $\begin{array}{l}\text { CR,PR,SD } \\
(n=52)\end{array}$ & $\begin{array}{l}\text { PD } \\
(n=15)\end{array}$ & \multirow[t]{2}{*}{$\begin{array}{l}\text { P- } \\
\text { value }\end{array}$} \\
\hline & & \multicolumn{2}{|l|}{ n $(\%)$} & \\
\hline \multicolumn{5}{|c|}{ Age (years) } \\
\hline$\leq 60$ & 43 & $35(81)$ & $8(19)$ & \multirow[t]{2}{*}{0.036} \\
\hline$>60$ & 24 & $17(70)$ & $7(30)$ & \\
\hline \multicolumn{5}{|l|}{ T stage } \\
\hline $\mathrm{T} 2$ & 19 & $14(74)$ & $5(26)$ & \multirow[t]{3}{*}{0.02} \\
\hline T3 & 34 & $30(88)$ & $3(12)$ & \\
\hline $\mathrm{T} 4$ & 14 & $8(57)$ & $6(43)$ & \\
\hline \multicolumn{5}{|c|}{ Smoking history } \\
\hline $\mathrm{No}$ & 8 & $8(100)$ & 0 & \multirow[t]{2}{*}{$<0.05$} \\
\hline Yes & 59 & $44(75)$ & $15(25)$ & \\
\hline
\end{tabular}

CR: Complete response; PR: Partial response; SD: Stable disease; PD: Progressive disease

All the evaluable 67 patients completed the scheduled 3 Cycles of NAC. The toxicity pattern is shown in table 3. Grade III and IV toxicities were infrequent and occurred in only $5 \%$ of patients. There was no chemotherapy-related mortality.

The most common haematological toxicity was anemia, mostly of grade I. Only 2 patients developed grade III anemia which was corrected with blood transfusion and the course of therapy was completed. Vomiting was universal in all cases with no grade IV vomiting reported. The only reported grade IV toxicity was nephrotoxicity in one patient which was corrected with haemodialysis and supportive measures.

After concluding NAC, we followed up the patients to know their attitude towards definitive treatment. All patients $(6,9 \%)$ who achieved pathologically confirmed CR by cystoscopic biopsy were shifted to bladder preservation treatment with radical radiotherapy. Of the 46 patients who had PR, $40(59.7 \%)$ patients underwent cystectomy. The other $6 \mathrm{PR}$ patients were satisfied with the results of the treatment (disappearance of symptoms) and they refused to have cystectomy, $3(4.5 \%)$ of them preferred radiotherapy and the remaining $3(4.5 \%)$ refused any form of definitive treatment in spite of our advice. The remaining $15(22.4 \%)$ patients had PD on treatment and were referred to radiotherapy, $2^{\text {nd }}$ line chemotherapy or salvage cystectomy.

In total, after completion of NAC, 9 (13.4\%) patients were shifted to bladder preservation treatment (according to patient preference) due to CR in 6 of them and major PR in the other 3 patients who refused cystectomy.

\section{DISCUSSION}

The dropout rate $(21 \%)$ in the current study is the usual rate in our center which serves a very wide geographic area covering nearly half of the Egyptian area. Hence, a patient may prefer to complete his treatment or his follow up in a nearby facility, after being offered the initial treatment, or the plan of treatment in our hospital.

Although considered as the state of the art in treatment of MIBC and being highly recommended in nearly every considerable guideline ${ }^{13-16}$, NAC in treatment of MIBC isn't widely practiced as recommended ${ }^{10}$. This may be attributed to the urologists' fear of missing the chance to operate on the patient in the "appropriate time" due to the duration needed for NAC and the possible side effects of the treatment.

In this study, we investigated the feasibility of NAC in MIBC, its side effects and how it affects the delivery of definitive treatment to the patients in our locality. We assigned our patients for 3 cycles of neo-adjuvant gemcitabine-cisplatin combination which is as effective as the standard methotrexate, vinblastine, doxorubicin, and cisplatin (MVAC) combination NAC with fewer side effects ${ }^{17}$.

Larger studies that investigated standard cisplatin based regimen confirmed the survival benefits of NAC in MIBC especially when $\mathrm{CR}$ is achieved ${ }^{13}$. The response rate in our study $(9 \% \mathrm{CR}, 61 \% \mathrm{PR}, 7.5 \% \mathrm{SD}$ and $22.4 \%$ PD) is very similar to single institute studies with small number of patients ${ }^{18}$. In an Egyptian Phase II study done by Khaled et al., 57 previously untreated patients with stage III/IV BC $(65 \%$ had transitional cell carcinoma) received neoadjuvant gemcitabine-cisplatin. The overall response rate was $59 \%$ with $9 \% \mathrm{CR}$ and $50 \%$ PR ${ }^{19}$. Galsky et al., reported an overall response rate of $56 \%$ in 25 patients treated sequentially with dose dense MVAC and gemcitabine ${ }^{20}$.

Overall, the gemcitabine-cisplatin regimen was well tolerated by our patients with grade III-IV toxicity in only $5 \%$ of patients. In a large randomized multicenter phase III study, gemcitabine-cisplatin was compared with MVAC. The trial revealed a similar efficacy with respect to response between the 2 treatment arms, whereas gemcitabine-cisplatin was significantly less toxic ${ }^{17}$. This is the foundation of using the "alternate regimen" instead of the "standard regimen" in our study. Herchenhorn et al used a similar regimen to ours and reported an incidence of $85 \%$ grade I toxicity, $28 \%$ grade II, $33 \%$ grade III and $4 \%$ grade IV ${ }^{18}$. Kaneko et al reported grade III-IV neutropenia in $14.3 \%$ of patients, anemia in $2.4 \%$ and thrombocytopenia in $21.4 \%$ of patients ${ }^{21}$, which is higher than we encountered in our study.

None of the patients included in this study died because of treatment-related cause. The NAC treatment related mortality is usually reported to be in the range of 0 to $1 \%{ }^{13,22}$. 
Table 3: Neoadjuvant chemotherapy - related acute toxicities

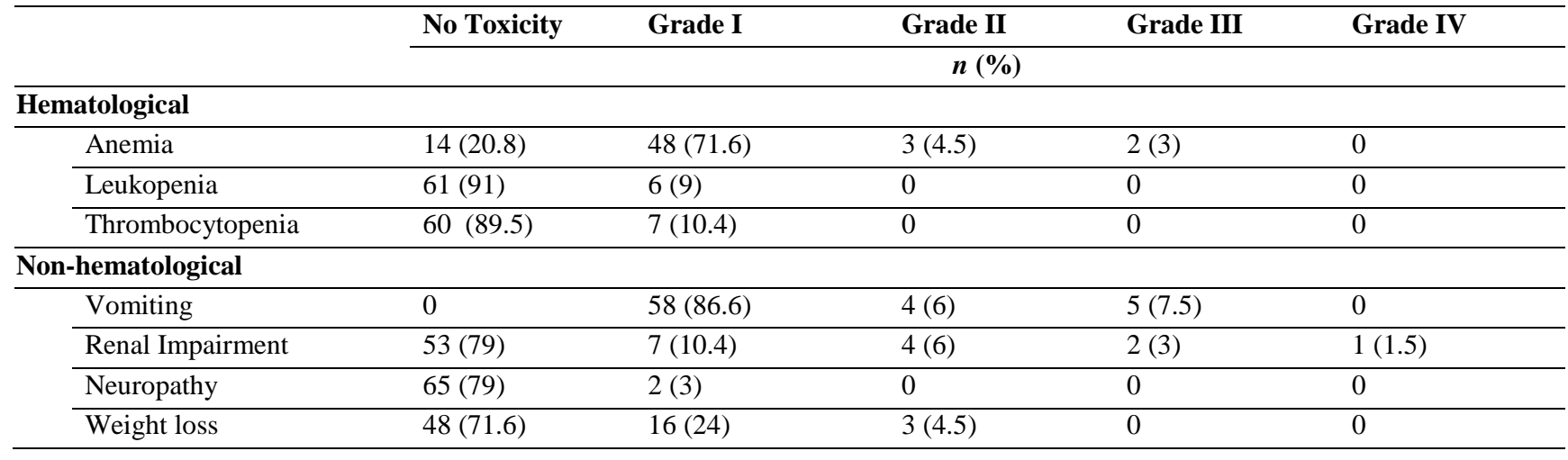

Since there are no tumor markers yet that can beforehand detect responders to NAC, certain clinicopathological factors were suggested to affect the treatment response. The relation between the response rate and stage of the $1^{\text {ry }}$ tumor was previously proved in a trial done by Schultz et al who reported overall response rates after neoadjuvant MVAC in patients with T3b and T4a tumors to be approximately $81 \%$ and $9 \%$, respectively ${ }^{23}$. In the present study the overall response rate in patients with T3 was $88 \%$ and in patients with T4 was $57 \%(p=0.02)$. In our study patient's age was significantly associated with response, as younger patients $(\leq 60$ years) responded better than older $(>60$ years) patients. Other researchers didn't find a significant difference between younger and older patients and concluded that any fit patient of any age group can benefit from the treatment ${ }^{24}$.

As regards smoking, Kim $\mathrm{PH}$ et al investigated the impact of smoking on the pathologic response to cisplatin-based NAC in patients with MIBC and found that smoking is not considered to be predictive of response ${ }^{25}$. On the contrary, in our study there was a significant association between smoking history and response to NAC which was better among non-smokers. Gender and grade were not found to be significantly affecting the response of NAC in the current study.

NAC does not prevent patients from undergoing cystectomy and does not increase the risk of perioperative complications. This was confirmed in a randomized controlled trial in which 317 patients with MIBC were randomized to radical cystectomy alone or 3 cycles of neoadjuvant MVAC followed by radical cystectomy. Cystectomy was performed as planned for $82 \%$ of patients assigned to NAC and $81 \%$ of those assigned to cystectomy alone ${ }^{22}$. This wasn't the case in our study. Only 9 (13.4\%) of our patients were shifted to bladder preservation due to $\mathrm{CR}$ in 6 patients in addition to another 3 who refused cystectomy after successful NAC treatment that resulted in disappearance of their symptoms due to major PR. This seems not to be unique to our patients. Herr reported on the outcome of 63 patients who refused cystectomy after receiving NAC for their MIBC ${ }^{5}$. We believe that multidisciplinary team management for BC patients and discussion of treatment options and expectations and better communication with the patient before starting treatment is necessary. It should be stressed on that NAC may result in CR, yet it does not mean a cure from MIBC without definitive local therapy. This can make the patients' expectations more realistic and improve the percentage of patients accepting cystectomy after NAC and hence, improving treatment outcomes.

\section{Conclusions}

Neoadjuvant chemotherapy is feasible to use with our patients as it gives good clinical response in the form of local control of the disease with tolerable side effects, and if offered in a proper way, it doesn't preclude the patients' chances for definitive treatment.

\section{REFERENCES}

1- Ibrahim AS, Khaled HM, Mikhail NN, Baraka H, Kamel $\mathrm{H}$. Cancer incidence in egypt: results of the national population-based cancer registry program. J Cancer Epidemiol. 2014; 2014: 437971.

2- Huang GJ, Stein JP. Open radical cystectomy with lymphadenectomy remains the treatment of choice for invasive bladder cancer. Curr Opin Urol. 2007; 17(5): 369-375.

3- Stein JP, Lieskovsky G, Cote R, et al. Radical cystectomy in the treatment of invasive bladder cancer: long-term results in 1,054 patients. J Clin Oncol. 2001; 19(3): 666675.

4- Honma I, Masumori N, Sato E, et al. Local recurrence after radical cystectomy for invasive bladder cancer: an analysis of predictive factors. Urology. 2004; 64(4): 744748.

5- Herr HW. Outcome of patients who refuse cystectomy after receiving neoadjuvant chemotherapy for muscleinvasive bladder cancer. Eur Urol. 2008; 54(1): 126-132.

6- Sonpavde G, Ross R, Powles T, et al. Novel agents for muscle-invasive and advanced urothelial cancer. BJU Int. 2008; 101(8): 937-943.

7- Herr HW, Bajorin DF, Scher HI. Neoadjuvant chemotherapy and bladder-sparing surgery for invasive bladder cancer: ten-year outcome. J Clin Oncol. 1998; 16(4): 1298-1301.

8- Advanced Bladder Cancer Overview Collaboration. Neoadjuvant chemotherapy for invasive bladder cancer. Cochrane Database Syst Rev. 2005; (2): CD005246.

9- Winquist E, Kirchner TS, Segal R, et al. Neoadjuvant chemotherapy for transitional cell carcinoma of the bladder: a systematic review and meta-analysis. J Urol. 2004; 171(2 Pt 1): 561-569.

10- Aragon-Ching JB. Neoadjuvant chemotherapy for muscleinvasive bladder cancer: are we asking the right questions? J Clin Oncol. 2014; 32(36): 4169-4170. 
11- Eisenhauer EA, Therasse P, Bogaerts J, et al. New response evaluation criteria in solid tumours: revised RECIST guideline (version 1.1). Eur J Cancer. 2009; 45(2): 228-247.

12- US Department of Health and Human Services. Common terminology criteria for adverse events (CTCAE) version 4.0. National Institutes of Health, National Cancer Institute. 2009; 4(03).

13- International Collaboration of Trialists; Medical Research Council Advanced Bladder Cancer Working Party (now the National Cancer Research Institute Bladder Cancer Clinical Studies Group); European Organisation for Research and Treatment of Cancer Genito-Urinary Tract Cancer Group; Australian Bladder Cancer Study Group; National Cancer Institute of Canada Clinical Trials Group; Finnbladder; Norwegian Bladder Cancer Study Group; Club Urologico Espanol de Tratamiento Oncologico Group, Griffiths G, Hall R, Sylvester R, Raghavan D, Parmar MK. International phase III trial assessing neoadjuvant cisplatin, methotrexate, and vinblastine chemotherapy for muscle-invasive bladder cancer: longterm results of the BA06 30894 trial. J Clin Oncol. 2011; 29(16): 2171-2177.

14- Alfred Witjes J, Lebret T, Compérat EM, et al. Updated 2016 EAU Guidelines on Muscle-invasive and Metastatic Bladder Cancer. Eur Urol. 2017; 71(3): 462-475.

15- National Comprehensive Cancer Network (NCCN) Clinical Practice Guidelines in Oncology. Bladder Cancer. Version 5, 2017. Available from: https://www.nccn.org/professionals/physician_gls/PDF/b1 adder.pdf

16- Bellmunt J, Orsola A, Leow JJ, Wiegel T, De Santis M, Horwich A; ESMO Guidelines Working Group. Bladder cancer: ESMO Practice Guidelines for diagnosis, treatment and follow-up. Ann Oncol. 2014; 25(Suppl 3): iii40-iii48.

17- von der Maase H, Hansen SW, Roberts JT, et al. Gemcitabine and cisplatin versus methotrexate, vinblastine, doxorubicin, and cisplatin in advanced or metastatic bladder cancer: results of a large, randomized, multinational, multicenter, phase III study. J Clin Oncol. 2000; 18(17): 3068-3077.

18- Herchenhorn D, Dienstmann R, Peixoto FA, et al. Phase II trial of neoadjuvant gemcitabine and cisplatin in patients with resectable bladder carcinoma. Int Braz J Urol. 2007; 33(5): 630-638.

19- Khaled H, Emara ME, Gaafar RM, et al. Primary chemotherapy with low-dose prolonged infusion gemcitabine and cisplatin in patients with bladder cancer: a Phase II trial. Urol Oncol. 2008; 26(2): 133-136.

20- Galsky MD, Iasonos A, Mironov S, Scattergood J, Boyle MG, Bajorin DF. Phase II trial of dose-dense doxorubicin plus gemcitabine followed by paclitaxel plus carboplatin in patients with advanced urothelial carcinoma and impaired renal function. Cancer. 2007; 109(3): 549-555.

21- Kaneko G, Kikuchi E, Matsumoto K, et al. Neoadjuvant gemcitabine plus cisplatin for muscle-invasive bladder cancer. Jpn J Clin Oncol. 2011; 41(7): 908-914.

22- Grossman HB, Natale RB, Tangen CM, et al. Neoadjuvant chemotherapy plus cystectomy compared with cystectomy alone for locally advanced bladder cancer. N Engl J Med. 2003; 349(9): 859-866.

23- Schultz PK, Herr HW, Zhang ZF, et al. Neoadjuvant chemotherapy for invasive bladder cancer: prognostic factors for survival of patients treated with M-VAC with 5-year follow-up. J Clin Oncol. 1994; 12(7): 1394-1401.

24- Chau C, Wheater M, Geldart T, Crabb SJ. Clinical outcomes following neoadjuvant cisplatin-based chemotherapy for bladder cancer in elderly compared with younger patients. Eur J Cancer Care (Engl). 2015; 24(2): 155-162.

25- Kim PH, Kent M, Zhao P, et al. The impact of smoking on pathologic response to neoadjuvant cisplatin-based chemotherapy in patients with muscle-invasive bladder cancer. World J Urol. 2014; 32(2): 453-459. 